\title{
Determination of reference physiological values for working donkeys of Ethiopia
}

\author{
Manyahilishal Etana ${ }^{1}$, Bojia Endeabu ${ }^{1}$, Shiferaw Jenberie ${ }^{2}$ and Haileleul Negussie ${ }^{3}$ \\ ${ }^{1}$ Donkey Health and Welfare Project Debre Zeit, P. O. Box. 34, Ethiopia \\ ${ }^{2}$ National Veterinary Institute, Debre Zeit, P. O. Box 19, Ethiopia \\ ${ }^{3}$ School of Veterinary Medicine, Addis Ababa University
}

\begin{abstract}
This study was undertaken with the aim of determining reference physiological values for working Ethiopian donkeys in four districts of Oromiya regional state by taking vital signs (body temperature, pulse and respiration rate) and gut sounds from a total of 130 apparently healthy donkeys. The mean values of body temperature obtained in this study for young, adult, and old donkeys were $37.90 \pm 0.74^{\circ} \mathrm{C}, 37.80 \pm 0.78^{\circ} \mathrm{C}$ and $37.70 \pm 0.63^{\circ} \mathrm{C}$, respectively. For female and male donkeys it was $37.88 \pm 0.79^{\circ} \mathrm{C}$ and $37.83 \pm 0.73^{\circ} \mathrm{C}$, respectively. The mean values of pulse rate per minute for young, adult and old donkeys were $55.40 \pm 9.93,47.60 \pm 5.47$ and 47.60 \pm 5.47 , respectively. For female and male donkeys it was $51.62 \pm 11.32$ and $49.69 \pm 8.59$, respectively. Pulse rate and heart rate had strong correlation $(r=0.824$; $\mathrm{p}<0.000$ ). The mean values of respiration rate per minute as determined by nostril

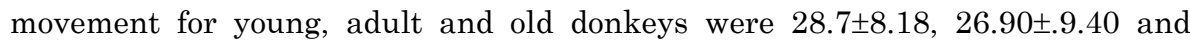
$28.40 \pm 7.79$, respectively. It was $28.40 \pm 9.84$ and $27.50 \pm 8.16$ for female and male donkeys, respectively. There was no statistically significant $(p>0.05)$ difference in nostril movement, abdominal movement and tracheal sound between daytime (morning Vs noon), age group and sex. However, strong and positive correlation $(\mathrm{r}=0.80 ; \mathrm{p}<0.000)$ was observed among the three sites of determining respiration. There was statistically significant difference $(p<0.000)$ in gut sounds heard at the right para-lumbar fossa (RPF), left para-lumbar fossa (LPF) and lower flank regions (LFR) during the times of day: more frequent and stronger at noon than in the morning. There was also a significant difference $(p<0.004)$ between young and old age groups in the value of RPF and LPF. The effect of age, sex and time of day on the references values should be taken into consideration in making physical clinical examination and interpreting physiological parameters.
\end{abstract}

Keywords: Equid, Ethiopia, Physiological, Working Donkeys, 


\section{Introduction}

Donkeys are one of the most important domestic animals most intimately associated with human. They contribute a lot through their involvement in different social and economic sectors. In Ethiopia donkeys have been used as a beast of burden for a long time and still render their valuable services, mostly as pack animals, throughout the country in general and in areas where modern means of transportation are absent, unaffordable or inaccessible in particular (Getachew Mulugeta et al., 2002). Despite their prominent role in agriculture based policy of the country, knowledge on physiology, nutritional requirements, health problems and management systems is limited except the attempts made by The Donkey Sanctuary since its inception (Starkey, 1997; Fielding, 1988). Hence, donkeys have low status in many of the regions where they are now found (Pearson and Merritt, 1991; de Aluja, 1998; Getachew Mulugeta, 1999).

Disease diagnosis in animals is largely dependent on clinical examination and laboratory results expected to reflect biological variations. This in turn requires understanding of the basal biophysical, hematological and serobiochemical profiles of an apparently healthy animal whose measurement provides invaluable information concerning the health status of an animal (Mori et al., 2003; Mori et al., 2004). In Ethiopia, few works have been attempted to determine normal physiological parameters of donkeys. However, with the exception of the work by Feseha Gebreab (1994) on some blood parameters, there has been no detailed investigation on the physiological parameters. Therefore, this study was conducted to determine reference physiological parameters of clinical importance for local working donkeys.

\section{Materials and Methods}

\section{Study area}

The study was conducted in 2005 at four central districts of Oromiya Regional State of Ethiopia, where the Donkey Health and Welfare Project (DHWP) has been operating for more than a decade; namely: Ada, Akaki, Dugda-Bora and Berhe district. The average daily maximum and minimum temperatures for Ada, Akaki, Dugda-Bora and Berhe district were $30.5^{\circ} \mathrm{C}$ and $8.5^{\circ} \mathrm{C}, 20^{\circ} \mathrm{C}$ and $18^{\circ} \mathrm{C}, 28^{\circ} \mathrm{C}$ and $22^{\circ} \mathrm{C}$ and $28^{\circ} \mathrm{C}$ and $15^{\circ} \mathrm{C}$, respectively (NMSA, 2004). 


\section{Study animals and sampling methods}

Apparently healthy and working donkeys were the sampling units for this study and all were reared under semi-intensive farming system; that means free to graze on pasture and rarely provided with other feed supplements mainly straw and coarse grain usually after strenuous work. Donkeys within the age range of 4 months to 20 years, as determined by dentition following description given by McCarthy (1986), were included in the study. Accordingly, study animals were grouped into three age categories: young (<2years), adult ( $2-15$ years) and old (>15 years). Moreover, all donkeys in the study had body condition score between 2 and 2.5 according to Svendsen (1997). Of the total 263 donkeys sampled randomly from the above four districts, 138 donkeys were selected for the study and of these $88(63.88 \%)$ were male and $50(36.12 \%)$ were female. These were selected based on body condition score (Svendsen, 1997).and clinical examination findings (mucous membrane, pregnancy status, dental examination, skin \& musculoskeletal conditions) and those donkeys with an abnormality of any type or pregnant were withdrawn from the study. Donkeys that had passed this first screening were followed for two weeks prior to determining physiological parameters. During this follow-up period, blood samples from ear vein for haemoparasites and faeces for parasitic eggs and larvae were analyzed. Donkeys were dewormed at the beginning of the follow-up period with ivermectin irrespective of the EPG count, and with triclabendazole when diagnosed positive for trematode. Yet eight (8) donkeys were excluded at this stage since they were diagnosed positive for parasite eggs at the end of the follow-up period and eventually 133 (50.6\%) donkeys were withdrawn. Only 130 donkeys were included in the study. These were $84(65 \%)$ male and $46(35 \%)$ female. Measurements were taken twice a day at 9:00 am and 3:00 pm for all donkeys in the study.

\section{Vital signs (Body temperature, pulse/heart rate and respiratory rate) determination}

Donkeys were examined clinically to determine vital signs and gut sound after being rested for a minimum of half an hour until all environmental stresses that might lead to physiological disturbances were reduced to the minimum. These reference physiological values were determined from the following site: pulse rate from submandibular artery, heart rate under the left side at the cardiac region, respiratory rate was assessed from three aspects: tracheal sound, number of nostril movements, and abdominal movement and finally rectal temperature was taken twice a day. 


\section{Gut sound determination}

Donkeys were examined to determine the gut sounds at the right paralumbar fossa (RPF) (right upper flank), left paralumbar fossa (LPF) (left upper flank) and lower flank region (LFR) (lower right and left flank). The recurrence or frequency as well as the strength of the gut sound heard at the above areas were assessed using stethoscope twice a day at morning and noon.

\section{Data analysis}

Data were analyzed using SPSS Version 15 software to determine the mean values of physiological body parameters and to assess the presence of significant difference in these values with regard to factors supposed to cause variation such as age, sex and daytime. Student's $t$-test, $95 \%$ confidence interval (CI) and $5 \%$ absolute precision were used throughout the study.

\section{Results}

The mean with its $95 \%$ confidence interval, standard deviation and median of physiological values for body temperature, heart, pulse and respiratory rates of apparently healthy donkeys are depicted in Table 1, 2 and 3 with respect to variation in age (young, adult and old), sex and daytime. There was a strong correlation $(\mathrm{r}=0.90 ; \mathrm{P}<0.001)$ between the mean body temperature readings or values of digital and mercury thermometers. Although the difference in body temperature was no statistically significant between adult and old donkeys, there was a statistically significant $(\mathrm{p}<0.05)$ difference in body temperature between young and old donkeys. The difference in body temperature between sexes and daytimes were not statistically significant $(p=0.08)$.

There was a strong correlation $(\mathrm{r}=0.824, \mathrm{p}<0.000)$ between pulse and heart rate. The difference in pulse and heart rate between young and the other two age groups was statistically significant $(p<0.05)$. However, there was no statistically significant difference $(\mathrm{p}>0.05)$ in heart and pulse rate between adult and old animals. Heart and pulse rates were not affected significantly ( $>0.05)$ by sex and daytime.

There was strong positive correlation $(\mathrm{r}>0.8 ; \mathrm{p}<0.000)$ among the three alternative methods of assessing respiration (tracheal sound, abdominal and nostril movement). The difference in respiration rate were not statistically significant $(p>0.05)$ between sexes. Although it was not significant $(p>0.05)$, noon time respiration were slightly higher than morning. Respiration in adult 
donkeys was significantly $(\mathrm{p}<0.05)$ lower compared to the other two age groups. There was also positive correlation $(r>0.6 ; p<0.05)$ among body temperature, pulse rate, heart rate and respiratory rate.

As shown in Table 4, there was statistically significant difference $(p<0.000)$ in gut sound heard at RPF and LPF during the morning and noon time: more frequent at noon than morning. Similarly, the strength of the gut sound heard at LFR was significantly $(p<0.05)$ higher at noon than in the morning. There was also a significant difference between young and old age groups in the value of RPF $(\mathrm{p}<0.004)$ and LPF $(\mathrm{P}<0.05)$. For the strength of gut sounds heard at LFR, there was no significant difference $(p>0.05)$ among the different age groups. There was no significant difference $(p>0.05)$ in the values of RPF, LPF and LFR between sexes.

\section{Discussion}

Unlike other domestic animals, research undertaken on donkey medicine, biology, physiology, management and socioeconomics are scarcely available in Ethiopia except the attempts made by animal charity group, The Donkey Sanctuary. Since there is insufficient data on physiological values of donkeys in Ethiopia, today veterinary clinicians who rarely treat donkeys have been using the physiological values of horses assuming that they have closer physiological features. Hence, the results of this study are compared with available research findings on donkey in other countries. The higher body temperature observed in young animals compared to older animals was in agreement with result reported by French and Patrick (1995). This high body temperature in young donkeys was attributed to their high metabolic rate (Fielding and Krause, 1998). A work conducted in the tropics, Ghana, by Canacoo et al., (1991) indicated that a mean morning and noon temperature values $\left({ }^{\circ} \mathrm{C}\right)$ of adult donkeys were $36.9 \pm 0.3$ and $37.8 \pm 0.2$, respectively. These findings are slightly lower than the results of the present study (37.9 \pm 0.84 and $37.8 \pm 0.81)$. This might be attributed to a relatively small sample size $(\mathrm{N}=27)$ used by Canacoo et al., (1991) compared to this study $(\mathrm{N}=130)$. The lower body temperature values in older animals and slightly higher mean body temperature in females in this study was in agreement with the report by Kelly (1977).

Currently in Ethiopia clinicians are using interchangeably mercury and digital thermometers to measure body temperature of their patients. However, there was no any attempt to see the correlation between the two types of thermometers. In this study strong positive correlation observed between the 
digital and mercury thermometer suggested that a clinician can use either of the two during physical clinical examination of donkeys. The median value for pulse rate (per minute) in this study in younger animals (56) was slightly lower than the result reported by French and Patrick (1995) (60). The mean morning pulse rate value for adult (47.85 \pm 9.33$)$ was slightly higher compared

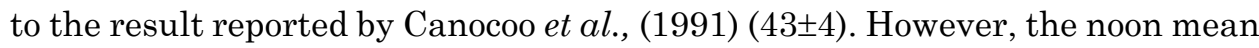
pulse rate (51 \pm 5$)$ reported by Canocoo et al., (1991) is slightly higher compared to the result of the present study (47.89 \pm 6.22$)$. These slight differences in pulse rate from the present study might be due to variations in environmental and geographical locations. The mean pulse rate value for females in this study is higher compared to males. This result is in agreement with the result reported by Canocoo et al., (1991) and the observations made by Kelly (1997) on most domestic animals. Strong correlation in nostril movement, tracheal sound and abdominal movement observed in this study was in agreement with Kelly (1997) which in turn indicated that a clinician can use any of these methods of determining respiratory rate.

Although it was not statistically significant $(p>0.05)$ there was a slight increase in respiratory rate at noon than morning. As reported by French and Patrick (1995), in this study significantly higher respiratory rate was observed in young age groups compared to adult donkeys. The positive correlation observed among temperature, heart rate, pulse rate and respiration rate indicated that as one of these parameters increase the others are also expected to rise. It has been shown that the overriding environmental factor affecting the body functions of domestic animals is temperature. In tropical and subtropical countries an animal may often be under heat stress when the temperature falls outside the comfort zone. In a hot humid climate, the ability of the air to absorb additional moisture is limited, and the inadequate cooling may result in heat stress. High body temperature initiates compensatory and adaptive mechanisms to re-establish the body homeostasis. Thus, physiologic responses of the animal during exposure to environmental stress have been determined by variations in body temperature, respiratory rate and heart rate. Changes in body temperature, respiratory rate and heart rate are used as indices of meteorological stress, whereas the absence of any change serves as an index of tolerance (Ayo et al., 1998).

Generally the recurrence time of gut sound heard at RPF and LPF was significantly $(p<0.05)$ shorter at noon than morning. Similarly the strength of the gut sound heard at LFR was significantly $(p<0.05)$ higher at noon than morning. The higher frequency and strength of the gut sound heard at noon 
was associated with the movement of ingesta from one part of the gut to the other and this is exactly in agreement with Pearson and Merritt (1991) and Svendsen (1997) who stated that gut sound is influenced by transient time of ingesta along the course of the gastrointestinal tract.

\section{Conclusions}

Equids particularly donkeys have been totally neglected despite their prominent role in both rural and urban society of the country. Hence, research on donkeys has lagged far behind other domestic species. Therefore, strengthening research to get maximum output from this species without violating its welfare requires urgency. In this study reference physiological values were determined for working donkeys in Ethiopia. This study indicated that considerable variations in physiological values attributed to variation in age, sex and daytime should be noted during physical clinical examination. The strong positive correlation with body temperature, heart, pulse and respiratory rate in this study implies that a rise in one of these physiological values leads to a rise in the other values. Although there are pioneer data on some of these parameters, the present findings contribute a lot to enhance our understanding on physiological parameters of Ethiopian donkeys; this will allow veterinarians and other practitioners to establish an appropriate interpretation of clinical findings and reach correct diagnosis of a disease condition in donkeys.

\section{References}

Ayo J. O, Oladele, S. B., Ngam, S., Fayomi, A. and Afolayan, S. B., 1998. Diurnal fluctuations in rectal temperature of the Red Sokoto goat during the harmattan season, Res. Vet. Sci., 66:7-9.

Canacoo, E. A., Eliis- Sackey, G. J. and Quashie, B. S., 1991. Some physiological values of apparently healthy donkeys in Ghana, Proceedings of the international Colloquium, Edinburgh, Scotland, $3^{\text {rd }}$ - 6th September, pp. 98-102.

de Aluja, A.S., 1998. The welfare of working Equids in Mexico, J. Appl. Anim. Welf. Sci., 59: 19-29.

Gebreab, F., 1994. Some blood parameters of Ethiopian working donkeys. In: working equines, Section 1: Health, Husbandry and Welfare, pp. 75-82.

Fielding, D., 1988. Reproductive characteristics of the Jenny Donkeys - Equus Asinus: A Review In Trop. Anim. Hlth. Prod., 20:161-166.

Ethiop. Vet. J., 2011, 15 (1), 79-86 
Fielding, D. and Krause, P., 1998. The tropical agriculturalist-donkeys. In: Coste, R., Smith, A.J., eds., London and Basingstoke, Macmillan Education Ltd; 119.

French, J.M. and Patrick, V.H., 1995. Reference values for physiological, hematological and biochemical parameters in domestic donkeys (Equus asinus), Equine Vet. Educ., 7:33-35.

Mulugeta, G., Gebreab, F., Trawford, F.A., Endeabu, B., Fanta, A. and Bayissa, A.., 2002. Some common clinical cases and interventions of the Donkeys Health and Welfare project, Donkey sanctuary Debre Zeit Ethiopia. In: Fourth international colloquium on working equines by Pearson, A., Fielding, D., Tabba, D., 2002. University Hama, Syria, pp. 96-109.

Mulugeta, G. ., 1999. Epidemological studies on the health and welfare of the Ethiopian donkeys with particular reference to parasite disease, MVM Thesis, Glasgow University.

Kelly, W. R., 1997. Veterinary Clinical Diagnosis, $3^{\text {rd }}$ Edition, Bailliere Tindall, London.

McCarthy, E., 1986. The principles and practices of feed rationing for donkeys. In: The professional Handbook of the Donkeys (Ed. Svendsen E.D.), Soveigh printing group, England, pp. 48-50.

Mori, E., Fernandes, W.R., Mirandola, R. M. S., Kubo, G., Ferreira, R. R., Oliveira, J. V. and Gacek, F., 2003. Reference Values on Serum Biochemical Parameters of Brazilian Donkey (Equus asinus) Breed, J. Equine Vet. Sci, 23: 356-364.

Mori, E., Mirandola, R.M.S., Ferreira, R.R., Oliveira, J.V., Gacek, F. and Fernandes, W.R., 2004. Reference Values on Hematologic Parameters of the Brazilian Donkey (Equus asinus) Breed, J. Equine Vet. Sci., 24:271-276

National Meteorology Service Agency (NMSA), 2004, Addis Ababa, Ethiopia.

Pearson, A. R. and Merritt, B.J., 1991. Intake, Digestion and Gastrointestinal transit time in resting donkeys and ponies and exercised donkeys given ad libitum hay and straw diets, Equine Vet. J., 23: 339-343.

Starkey, P., 1997. The professional Hand Book of the Donkey. In: Svendsen, E.D. London, $3^{\text {rd }}$ ed, pp.108-110.

Svendsen, E. D., 1997. Parasites Abroad. In: Svendsen E.D. (ed). The Professional Hand Book of the Donkey. $3^{\text {rd }}$ edition, Whittet books, London, pp. 227-238. 